\title{
Automated cystic mass extraction from ultrasound phantom images
}

\begin{abstract}
The aim of this work is to automatically extract Cystic Masses from Ultrasound Phantom images and improve the efficiency of interpretation using Computer-Aided Detection. To make it a general algorithm, 6 most popular ultrasound machines were selected and following parameters were swept: modes of operation, transducer, frequency and contrast, while making phantom images. Ultrasound images were acquired using a quality multi tissue Ultrasound Phantom in B-Mode. Gamma corrections, contrast stretching, filtering and morphological Image Processing were among the steps that were applied to find the output image. Two experienced radiologists marked final images. Statistical analysis of results showed a sensitivity of $99 \%$ and accuracy of $98 \%$ for proposed framework. As a side result based on the actual depth of each image, processing time were also decreased.
\end{abstract}

Keyword: Computer aided detection; Ultrasound; Medical image processing; Breast cancer; Ultrasound phantom; Digital image processing 